\title{
Mixed-transform based codec for 2D compression of ECG signals
}

\author{
Johan Chagnon and Laura Rebollo-Neira \\ Mathematics Department \\ Aston University \\ B3 7ET, Birmingham, UK
}

\begin{abstract}
A method for ECG compression, by imaging the record as a $2 \mathrm{D}$ array and implementing a transform lossy compression strategy, is advanced. The particularity of the proposed transformation consists in applying a Discrete Wavelet Transform along one of the dimensions and the Discrete Cosine Transform along the other dimension. The performance of the method is demonstrated on the MITBIH Arrhythmia database. Significant improvements upon the 1D version of the codec, and on benchmarks for 2D ECG compression, are achieved.
\end{abstract}

\section{INTRODUCTION}

Cardiovascular diseases (CVDs) are the number one cause of global death. The World Hearth Organization has estimated that 17.9 million people died from CVDs in 2016. Over three quarters of these deaths take place in low and middle income countries. A major goal in the Sustainable Development Agenda of the United Nations is to reduce these figures one third by 2030. Within this agenda, prevention and routine controls play a central role. The electrocardiogram (ECG) is one of the most common tests in the diagnosis of CVDs. It goes without saying that techniques for safely compressing these types of data are essential to the development and support of clinical health care.

In a recent publication [1] a method for effective high compression of ECG signals has been proposed. That method, which is applied on a 1D record, was shown to significantly improve upon benchmarks on the same database [2]-[4]. In this Communication we extend the compression technique in [1] to allow for its application on a 2D array constructed out of 1D ECG signal. It is demonstrated that, for the same level of distortion, the average compression performance on the MIT-BIH Arrhythmia database considerably improves in relation to the $1 \mathrm{D}$ processing. The method is also shown to improve upon previous state of the art benchmarks concerning 2D ECG compression [5]. MATLAB software for reproducing results and facilitating future comparisons has been made available on a dedicated webpage [6].

\section{Proposed Coding Strategy}

A digital ECG signal represents a sequence of heartbeats, each of which is characterized by a combination of three graphical deflections, known as QRS complex, and the so called $\mathrm{P}$ and $\mathrm{T}$ waves. 2D ECG compression relies on this feature. Our approach operates on raw data and consists of the following steps.

A) $1 \mathrm{D}$ to $2 \mathrm{D}$ conversion by segmentation and alignment of heartbeats.

B) Application of a Discrete Wavelet Transform (DWT) along the direction of the segmented beats and the Discrete Cosine Transform (DCT) along the perpendicular direction.

C) Quantization, organization, and entropy coding of the information needed to recover the ECG record from the compressed file.

\section{A. $1 D$ to $2 D$ conversion}

The conversion of the 1D ECG record into a 2D array requires segmentation and alignment of heartbeats. We implement this step as done in [5]. Firstly a QRS detection algorithm is applied to locate the R peaks. Each of these peaks is aligned with the previous one. Since the length of the heartbeats are not uniform, a regular array $\mathbf{A}$ is obtained by padding rows with zeros. The duration of the heartbeats are stored as components of a vector, say $\mathbf{h}$, which has to be passed on to the decoder. Fig 1 illustrates a $2 \mathrm{D}$ array of size $86 \times 359$ produced from a short $1 \mathrm{D}$ ECG record consisting of 25,000 samples.

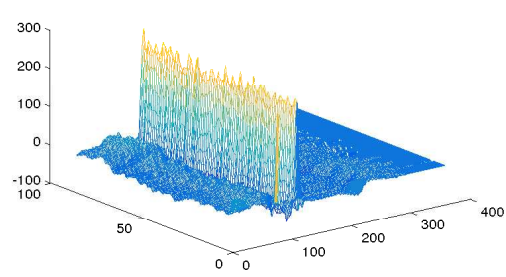

Fig. 1. 2D array produced by segmentation and alignment of heartbeats.

\section{B. Transformation of the $2 D$ array}

This step introduces the distinctive feature of the proposed codec. Instead of applying a 2D wavelet transform 
on the array $\mathbf{A}$, as it is done in other 2D ECG compression methods, e. g. [5], we apply a different transform in each dimension. More precisely, we apply the 1D cdf97 DWT on the rows of $\mathbf{A}$ and the 1D DCT transform on the columns of $\mathbf{A}$, i.e., we create the transformed array $\mathbf{B}$ as follow:

$$
\mathbf{B}=\widehat{\mathcal{W}}_{1 r} \widehat{\mathcal{C}}_{1 c} \mathbf{A},
$$

where $\widehat{\mathcal{W}}_{1 r}$ indicates the $1 \mathrm{D}$ cdf97 DWT operating on the rows of the array $\widehat{\mathcal{C}}_{1 c} \mathrm{~A}$ and $\widehat{\mathcal{C}}_{1 c}$ indicates the DCT transform operating on the columns of the array $\mathbf{A}$. The convenience of performing this mixed transformation for encoding purposes becomes clear from the graphs of Fig. 2. The upper graph represents the absolute value of the 2D cdf97 DWT of A and the lower graph the absolute value of the array $\mathbf{B}$ as given in (1). The distribution of most significant elements in $\mathbf{B}$ (lightest regions in the lower graph) benefits the coding strategy described below.
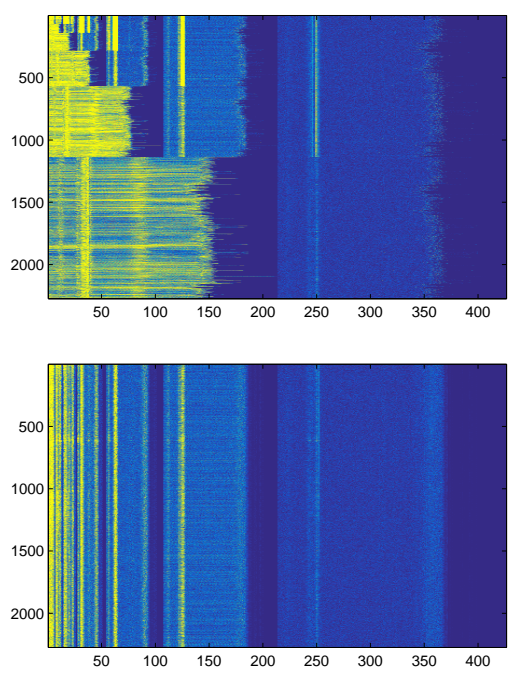

Fig. 2. Magnitude of the 2D cdf97 DWT (upper graph) and magnitude of mixed transform given in (1) (lower graph).

\section{Encoding}

The encoding process begins by adopting a columnmajor order to express the $N \times M$ array $\mathbf{B}$ as a $1 \mathrm{D}$ vector $\mathbf{b}=(b(1), \ldots, b(N M))$. Hereafter the encoding proceeds as in [1]. The components of $\mathbf{b}$ are converted to integer numbers by a mid-tread uniform quantizer as follows:

$$
b^{\Delta}(i)=\left\lfloor\frac{b(i)}{\Delta}+\frac{1}{2}\right\rfloor, \quad i=1, \ldots, N M .
$$

where $\lfloor x\rfloor$ indicates the largest integer number smaller or equal to $x$ and $\Delta$ is the quantization parameter.

The absolute value of the elements (2) are placed in a smaller vector, say $\mathbf{c}=(c(1), \ldots, c(K))$, after the elimination of zeros. The signs are encoded separately in a vector $\mathbf{s}=(s(1), \ldots, s(K))$ using a binary alphabet $(1$ for + and 0 for - ).
Assuming that the nonzero values in (2) occur at the positions $\ell_{i}, \ldots, \ell_{K}$, these indices are re-ordered in ascending order $\ell_{i} \rightarrow \tilde{\ell}_{i}, i=1, \ldots, K$, which guarantees that $\tilde{\ell}_{i}<\tilde{\ell}_{i+1}, i=1, \ldots, K$. This induces a re-order in the coefficients, $\mathbf{c} \rightarrow \tilde{\mathbf{c}}$ and in the corresponding signs $\mathbf{s} \rightarrow \tilde{\mathbf{s}}$. Defining $\delta(i)=\tilde{\ell}_{i}-\tilde{\ell}_{i-1}, i=2, \ldots, K$ the array $\boldsymbol{\delta}=\left(\tilde{\ell}_{1}, \delta(2), \ldots, \delta(K)\right)$ stores the indices $\tilde{\ell}_{1}, \ldots, \tilde{\ell}_{K}$ with unique recovery.

Finally the vectors $\tilde{\mathbf{c}}, \tilde{\mathbf{s}}, \boldsymbol{\delta}$, as well as the length of the heartbeats $\mathbf{h}$, are compressed using Huffman coding. The additional numbers which have to be passed to the decoder are: i) the quantization parameter $\Delta$ ii) the mean value of the $1 \mathrm{D}$ ECG record and iii) the size of the $2 \mathrm{D}$ array $\mathbf{A}$.

\section{1D ECG SIGNAL RECOVERY}

At the decoding stage, after reverting Huffman coding, the locations $\tilde{\ell}_{1}, \ldots, \tilde{\ell}_{K}$ of the nonzero entries in the transformed array, after quantization, are readily obtained. This allows the recovery of the array as follows. i) Set $b^{r}(i)=0, i=1, \ldots, N M$ and $b^{r}\left(\tilde{\ell}_{i}\right)=(2 \tilde{s}(i)-$ 1) $\tilde{c}(i) \Delta, i=1, \ldots, K$. ii) Reshape the vector $\mathbf{b}^{r}$ as a $2 \mathrm{D}$ array $\mathbf{B}^{r}$ of size $N \times M$. The array $\mathbf{A}^{r}$ is recovered from $\mathbf{B}^{r}$ inverting the $\widehat{\mathcal{W}}_{1 r}$ and $\widehat{\mathcal{C}}_{1 c}$ transformations (c.f.(1)). The re-conversion to the 1D signal say, $\mathrm{f}^{\mathrm{r}}$, from the $2 \mathrm{D}$ array $\mathbf{A}^{r}$ is straightforward using the heartbeat lengths in $\mathbf{h}$ and the mean value signal.

The achieved compression ratio $\mathrm{CR}$, which is defined as

$$
\mathrm{CR}=\frac{\text { Size of the uncompressed file. }}{\text { Size of the compressed file }}
$$

depends on the required quality of the recovered signal. This is assessed with respect to the standard PRD metric as given by

$$
\operatorname{PRD}=\frac{\left\|\mathbf{f}-\mathbf{f}^{\mathrm{r}}\right\|}{\|\mathbf{f}\|} \times 100 \%,
$$

where, $\mathbf{f}$ is the original signal, $\mathbf{f}^{\mathrm{r}}$ is the signal reconstructed from the compressed file and $\|\cdot\|$ indicates the 2-norm.

\section{Numerical RESUlts}

For all the tests the full MIT-BIH Arrhythmia database [7], which contains 48 ECG records, is used. Each of these records consists of $N=650,000$ 11-bit samples at a frequency of $360 \mathrm{~Hz}$. At the QRS detection step a MATLAB implementation [8] of the Pan Tompkins algorithm was applied [9].

The comparison with 1D compression is realized with respect to the 1D version of the strategy adopted here, which has been shown in [1] to over-perform previously reported results [2]-[4]. Table I produces comparisons for PRD in the range [0.4 1]. The range [0.4 0.7] corresponds to very low level distortion of the recovered signal. For PRD $<0.4$ the proposed coding strategy is not effective. Nonetheless, it is worth noting that, because the approach is applied on raw data, requiring PRD $<0.4$ implies to force the reproduction of the small high frequency 
noise present in all the records. As discussed in [1], the decomposition of the cdf97 DWT in 4 levels produces the best 1D compression results on the MIT-BIH Arrhythmia database. In 2D, however, results improve by decomposing into 6 levels. Table I shows the mean value CR and corresponding standard deviation (std) yielded by the 1D and 2D methods. In both cases the quantization parameter has a different value for each record so as to achieve, for every record in the database, the sharp values of PRD given in the first row of Table II Since the compressibility of the records is not uniform, fixing the same PRD for all the records generates large dispersion in CR. This is reflected in the std values.

TABLE I

MEAN VALUE COMPRESSION RATIOS FOR PRD VALUES SHARPLY REPRODUCED BY EVERY RECORD IN THE DATABASE.

\begin{tabular}{||l||r|r|r|r|r|r|r||}
\hline \hline PRD & 1.00 & 0.90 & 0.80 & 0.70 & 0.60 & 0.50 & 0.40 \\
\hline CR (1D) & 42 & 39 & 35 & 32 & 28 & 24 & 19 \\
std & 12 & 11 & 10 & 9 & 8 & 7 & 5 \\
CR (2D) & 85 & 73 & 61 & 50 & 39 & 29 & 20 \\
std & 50 & 42 & 34 & 28 & 21 & 15 & 10 \\
\hline \hline
\end{tabular}

Table [II produces comparison with results in Table VIII of [5], for the whole database with reconstruction quality in our range of interest. The method in [5] employs a modified set partitioning in hierarchical trees (SPIHT) algorithm, which is shown to produce superior results than other image compressions techniques, including JPEG2000. The reported values of PRD are said to have been calculated after subtraction of a baseline of 1024 to the original data. The corresponding values are indicated here as $\mathrm{PRD}_{\mathrm{B}}$. The notation PRD is kept to indicate the PRD with respect to raw data.

\section{TABLE II}

COMPARISON WITH THE RESULTS IN TABLE VII OF [5] FOR THE WHOLE DATABASE

\begin{tabular}{||c||c|c|c|c|c|c||}
\hline \hline Method & PRD $_{\mathrm{B}}$ & std & CR & std & PRD & std \\
\hline |5] & 6.82 & - & 30 & - & - & - \\
1D & 6.82 & 0.01 & 31 & 14 & 0.77 & 0.41 \\
2D & 6.82 & 0.01 & 58 & 63 & 0.77 & 0.41 \\
\hline $\mid 5]$ & 3.81 & - & 20 & - & - & - \\
1D & 3.81 & 0.01 & 19 & 10 & 0.43 & 0.23 \\
2D & 3.81 & 0.01 & 26 & 30 & 0.43 & 0.23 \\
\hline \hline
\end{tabular}

\section{REMARKS AND CONCLUSIONS}

The numerical tests demonstrate that the extension to operate in $2 \mathrm{D}$ of the $1 \mathrm{D}$ compression strategy proposed in [1] yields, on the whole, very significant improvement in compression power. A distinctive feature of the 2D approach is that, fixing quality, there is very large dispersion in the values of CR. This is due to the fact that compressing in $2 \mathrm{D}$ is greatly beneficial for records of very regular morphology. Only some of the records in the MIT-BIH Arrhythmia database possess this trait. Fig. 3 shows the histogram of $\mathrm{CR}$ for the $2 \mathrm{D}$ approach and $\mathrm{PRD}_{\mathrm{B}}=6.82$.

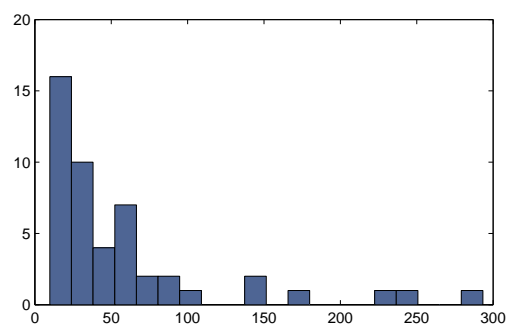

Fig. 3. Histogram of the CR obtained with the $2 \mathrm{D}$ approach corresponding to $\mathrm{PRD}_{\mathrm{B}}=6.82$ for every record in the database.

We are aware that the calculation of PRD subtracting a baseline of 1024 adopted in [5] has generated confusion leading to propagation of unfair comparison with values of PRD without subtraction of baseline. Table II gives the two metrics. The quantization parameter $\Delta$, which controls quality, has been set differently for each record in oder to reproduce the sharp values of $\mathrm{PRD}_{\mathrm{B}}$ as those reported in [5]. The values of std are not reported in that publication.

The benefit of $2 \mathrm{D}$ compression comes at the expense of some additional computation. With respect to the 1D implementation there is an extra time for QRS detection and for converting the 1D array into a 2D one. Furthermore, the Huffman coding step becomes more relevant in 2D compression than it is in 1D. However, as shown in Tables 【 and [1 the improvements in compression results justify the computational overhead.

\section{REFERENCES}

[1] L. Rebollo-Neira, "Effective high compression of ECG signals at low level distortion", Scientific Reports, 9, No 4564 (2019).

[2] S.J. Lee, J. Kim, and M. Lee, "A Real-Time ECG Data Compression and Transmission Algorithm for an e-Health Device", IEEE Trans. on Biomedical Engineering, 58, 2448 - 2455 (2011).

[3] J.L. Ma, T.T. Zhang, and M. C. Dong, "A Novel ECG Data Compression Method Using Adaptive Fourier Decomposition With Security Guarantee in e-Health Applications", IEEE Journal of Biomedical and Health Informatics, 19, 986 - 994 (2015).

[4] C. Tan, L. Zhang and H. Wu,"A Novel Blaschke Unwinding Adaptive Fourier Decomposition based Signal Compression Algorithm with Application on ECG Signals", IEEE Journal of Biomedical and Health Informatics 23, pp 672 - 682 (2018).

[5] S-C Tai, C-C Sun, and W-G Yan, "A 2-D ECG Compression Method Based on Wavelet Transform and Modified SPIHT", IEEE Trans. on Biomedical Engineering, 52, pp 999 - 1008 (2005).

[6] http://www.nonlinear-approx.info/examples/ node $014 . h t m l$

[7] https://physionet.org/physiobank/database/ mitdb/

[8] H. Sedghamiz, "Matlab Implementation of Pan Tompkins ECG QRS detector"., https://www.researchgate.net/publication/ 313673153_Matlab_Implementation_of_Pan_Tompkins_ECG_QRS detect (2014).

[9] J. Pan and W.J. Tompkins, "A Real-Time QRS Detection Algorithm”, IEEE Trans. on Biomedical Engineering, BME-32,pp 230236 (1985). 